\title{
Erratum to: Simulink to UPPAAL Statistical Model Checker: Analyzing Automotive Industrial Systems
}

\author{
Predrag Filipovikj ${ }^{1(\bowtie)}$, Nesredin Mahmud ${ }^{1}$, Raluca Marinescu ${ }^{1}$, \\ Cristina Seceleanu ${ }^{1}$, Oscar Ljungkrantz ${ }^{2}$, and Henrik Lönn ${ }^{2}$ \\ ${ }^{1}$ Mälardalen University, Västerås, Sweden \\ \{predrag. filipovikj, nesredin. mahmud, raluca. marinescu, \\ cristina.seceleanu\}@mdh.se \\ ${ }^{2}$ Volvo Group Trucks Technology, Gothenburg, Sweden \\ \{oscar.1jungkrantz, henrik.lonn\}@volvo.com
}

\section{Erratum to:}

\section{Chapter "Simulink to UPPAAL Statistical Model Checker:} Analyzing Automotive Industrial Systems" in:

\section{J. Fitzgerald et al. (Eds.): FM 2016: Formal Methods, LNCS, DOI: 10.1007/978-3-319-48989-6_46}

The initially published version of Figure $1 \mathrm{a}$ and $\mathrm{b}$ is incorrect. This is the correct version.

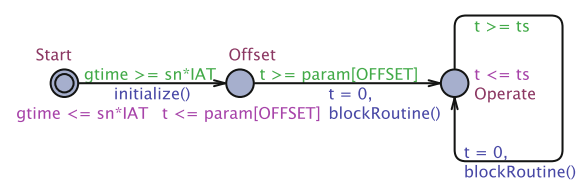

(a) Pattern for discrete blocks

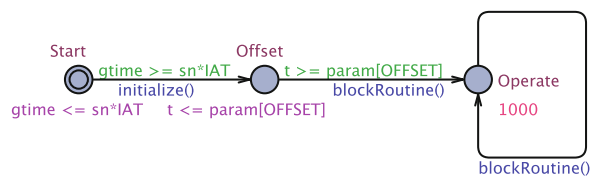

(b) Pattern for continuous blocks

Fig. 1. Our used TA patterns

\footnotetext{
The updated original online version for this chapter can be found at DOI: 10.1007/978-3-319-48989-6_46
} 